\title{
Transversus abdominis plane block: a new gold standard for abdominal surgery?
}

\begin{abstract}
Transversus abdominis plane (TAP) block is a technique of peripheral nerve block for abdominal anterior wall and in past years several authors demonstrated its efficacy and role in multimodal analgesia for various surgeries. Introduction of ultrasound-guided technique provided to more safety and easiness to realize this block after an adequate training, with description of few complications. Scarcity of contraindications makes this technique applicable in a large number of patients, whereas epidural analgesia is not recommended. Evolution of this block, with the possibility of using continuous infusion catheters, allows a longer and more effective control of postoperative pain after abdominal surgeries, with less respiratory and cardiovascular complications, a more comfortable recovery of patients and a consequent reduction of length hospital stay and costs, in line to Enhanced Recovery After Surgery (ERAS) pathways. Promotion and application of TAP block are to be encouraged in daily clinical practice to meliorate pain control, patient satisfaction and a fast recovery.
\end{abstract}

Volume 4 Issue 3 - 2016

\author{
Federico Fiorini,' Flaminio Sessa,' Elisabetta \\ Congedo, ${ }^{2}$ Germano De Cosmo \\ 'Department of Anaesthesiology and Intensive Care, University \\ Hospital “A. Gemelli”, Catholic University of Sacred Heart, Italy \\ ${ }^{2}$ Antonio Perrino Hospital, Italy
}

Correspondence: Germano De Cosmo, Department of Anaesthesiology and Intensive Care, University Hospital $\square$ A Gemelli, Catholic University of Sacred Heart, Rome, Italy, Tel 0635031, Fax063050101, Email gdecosmo@rm.unicatt.it

Received: January 28, 2016 | Published: February 12, 2016

Keywords: transversus abdominis plane block, thoracic epidural analgesia, patientcontrolled analgesia, enhanced recovery after surgery

Abbreviations: TAP, transversus abdominis plane; TEA, thoracic epidural analgesia; ERAS, enhanced recovery after surgery; US-Guided, ultrasound-guided; PCA, patient controlled analgesia

\section{Introduction}

Fifteen years have now elapsed since the first publication appeared on Transversus Abdominis Plane (TAP) block, by Rafi et al. ${ }^{1}$ it has described as the deposition of local anaesthetic drug in the neurovascular plane sited between transversus muscle and internal oblique muscle in correspondence of lumbar triangle of Petit, the anatomical space formed by the margin of the latissimus dorsi posteriorly, the margin of the external oblique anteriorly and the iliac crest inferiorly. To 200 patients undergoing to abdominal surgeries, in lateral position, he applied a blind technique to individuate this virtual space, through the "double-pop technique": the needle insertion in this area is guided by felting of the first pop, for the passage from external to internal oblique muscle, and the second pop, from the internal oblique to transversus abdominis muscle; a volume of $20 \mathrm{ml}$ of anaesthetic is deposited into this space after induction of anaesthesia and prior to surgery, obtaining a good control of pain in children and adult patients.

Subsequent studies, first on cadaveric anatomy then in volunteers, confirmed the importance of transversus abdominis plane and his function. In this space lateral cutaneous branches of intercostal, subcostal and first lumbar nerves that contribute to innervation of abdominal anterior wall run with their accompanying blood vessels; particularly, segmental nerves T6 to T9 emerged from the costal margin to enter the TAP, between the midline and the anterior axillary line, T6 entered the TAP just lateral to the linea alba, while T7, T8, and T9 emerged from the costal margin at increasingly lateral positions respectively, T9-L1 emerged medial or lateral to the anterior axillary line, with many intercommunicate branches; all branches ended to rectus sheath, under the rectus abdominis muscle, bilaterally to linea alba. ${ }^{2}$ These anatomic specifications are fundamental to understand real distribution of anaesthetics and the application of TAP block to reduce only the abdominal wall pain, not the visceral pain, like epidural analgesia does.
The introduction of ultrasound (US) guide in anaesthesia had permitted an indirect vision of internal structure (muscle, vessels, nerves): ultrasonography became an indispensable tool for anaesthesiologist and the gold standard for truncal and peripheral blocks, such as several international guidelines recommend. Authors started to describe ultrasound (US) guided TAP block, first more anterior respect to Petit triangle, between iliac crest and subcostal margin via an in-plane approach, in the mid-axillary line and with patient in supine position: continuous tip visualization during the procedure provided to identify muscle layers and planes, reducing wrong intramuscular distribution of anaesthetics and avoiding complications as peritoneum, bowel, liver and vessels puncture. ${ }^{3,4}$ For its fast and simple execution after a relatively short training period, few complication and effectiveness, this approach of unilateral or bilateral TAP block was used in last year's for many types of laparotomic, and then laparoscopic, surgeries with a great success: McDonnell applied US-guided TAP block for 16 laparotomic bowel resections with mid-line incisions and demonstrated a reduction of $70 \%$ morphine consumption and pain decrease in postoperative days respect a control group without TAP block; ${ }^{5}$ Niraj et al. ${ }^{6}$ showed analogue results with TAP block after open appendectomy, ${ }^{6}$ but also in laparotomic and laparoscopic hernia repair surgeries TAP block is effective to reduce postoperative pain, ${ }^{7,8}$ Caesarean section, ${ }^{9}$ laparoscopic colorectal surgeries, ${ }^{10,11}$ hysterectomies, ${ }^{12}$ open prostatectomy, ${ }^{13}$ laparoscopic hand-assisted nefrectomy, ${ }^{14}$ after renal transplantation, ${ }^{15}$ until robotic surgeries. ${ }^{16}$

Most of this authors introduced use of catheters in TAP, to continuous anaesthetic release, through a Tuohy needle inserted with the same in-plane technique: they allows a pain control for more than first 8-24 hours after surgeries, but also for two or three postoperative days, with a relevant opioid sparing and morphinerelated complications reduction. ${ }^{17,18}$

Moreover, recent studies demonstrated that this block can be realized in patients of all ages, from neonatal period, as showed by Fredrickson and Seal $\mathrm{P}^{19}$ for minor and major surgery with minor morphine consumption and faster recovery, until elderly patients, like suggested by Sammons and Ritchey $\mathrm{W}^{20}$ with an improved 
pain management and reduced incidence of postoperative delirium, pneumonia, urinary retention, and falls $;{ }^{20}$ certainly, these patients may benefit more from this technique, thanks to less use of postoperative opioid.

\section{One or many TAP blocks?}

In last years, authors showed an increasingly interest to TAP block: several studies of anaesthetic drug distribution in TAP were realized with the US guided technique and puncture above the iliac crest (the so called "lateral approach"), demonstrating that lateral TAP block does not reduce pain of any type of abdominal incision. Hebbard ${ }^{21}$ described a new approach, the "subcostal TAP block" ${ }^{21}$ the needle is inserted inplane from anteromedial to inferolateral with local injection between the rectus abdominis and transversus abdominis, more medial than anterior axillary line, whereas, anatomically, cutaneous branches of nerve T6 and T7 emerges. This type of TAP block can cover upper abdominal incision, corresponding to dermatomes T6-T9, ${ }^{22,23}$ from xyphoid process until umbilicus, while lateral TAP US-guided block covered area above umbilicus, corresponding to dermatomes T10-L1, like a cadaveric study showed. ${ }^{24}$

Subcostal TAP block is applied for laparoscopic cholecystectomy, where ensure a longer pain reduction rather than lateral TAP block, with a minor opioid use, ${ }^{25,26}$ for gastric resection, ${ }^{27}$ gastric bypass, ${ }^{28}$ after liver transplant ${ }^{29}$ and resection: ${ }^{30}$ in this last surgery, a study showed the execution of TAP block under the direct vision of anatomical space by surgeon during closure of inverted-L shaped incision, combined with PCA; for laparoscopic cholecystectomy, another work demonstrated relation of TAP block, reduction of postoperative pain and a better patients respiratory function. ${ }^{31} \mathrm{~A}$ MRI study compared local anaesthetic diffusion in volunteer between subcostal and in addiction with lateral TAP block, demonstrating more widespread dermatome anaesthesia on the hemi abdomen where the dual injections were performed, from T6 to T12, while a local spread from T10 to T12 for the lateral TAP alone. ${ }^{32}$

In this last years, some authors proposed a subdivision of subcostal approach in upper and lower subcostal TAP block, whereas the first one is the classical subcostal TAP block and covers mostly T6-T8 dermatomes, while the second one covers T7-T8 and US-guided puncture point is subcostal, but among the anterior and the midaxillary line: ${ }^{33}$ in a recent article, Takimoto et al..$^{34}$ compared two groups of patients undergoing laparoscopic cholecystectomy treated with lateral TAP block and adding double subcostal TAP block, with same results in term of postoperative pain. ${ }^{34}$

Another approach is the "posterior" TAP block, that remarks the classical landmark of Petit Triangle: with probe on the iliac crest, in correspondence of mid-axillary line, muscle layers are visualized and, with an in-plane technique, local anaesthetic are deposited in the area defined by the aponeurosis of the transversus abdomen is and the anterolateral-most part of the quadratus lumborum: the needle tip's location posterior to the fascia transversal is allows more direct spread of local anesthetic solution to the paravertebral space. Comparing with subcostal TAP block on 50 patients undergoing major abdominal surgery, Lee showed that the posterior approach produced a median sensory block of three dermatomal segments, from T10 to T12 and subcostal covered T7-T9 dermatomes..$^{35}$

A complete work based on MRI imaging compared this four approach of TAP block (subcostal, mid-axillary, anterior and posterior) on four groups of volunteer: anterior subcostal and midaxillary ultrasound approaches resulted in a predominantly anterior spread of the contrast solution within the transversus abdomen is plane and relatively little posterior spread; there was no spread to the paravertebral space with the anterior subcostal approach, the mid-axillary transversus abdomen is plane block gave faint contrast enhancement in the paravertebral space at T12-L2. In contrast, the posterior approaches, using both landmark and ultrasound identifications, resulted in predominantly posterior spread of contrast around the quadratus lumborum to the paravertebral space from T5 to L1 vertebral levels. ${ }^{36}$

Some authors identified a fifth approach of TAP block, considering the US guided block of ilio-inguinal and ilio-hypogastric nerve in TAP for appendectomy, ${ }^{32}$ medially to iliac crest. All these studies show that TAP block is a technique in constant evolution and innovation and more works are necessary to establish the superiority and the efficacy of one approach rather than another to treat postoperative pain in different surgeries.

\section{TAP block and local anaesthetic drugs}

Local diffusion into the TAP requires a large volume of anaesthetic to determine the effective spread on interested nerves: all authors suggested a median volume of $15-20 \mathrm{ml}$ of anaesthetic for each puncture. ${ }^{37}$ Although one may assume that a higher concentration of a local anaesthetic given via TAP block may provide better-quality postoperative analgesia, this raises the issue of potentially toxic plasma concentrations of the local anaesthetic, as the TAP block generally involves injection of a single large dose of local anaesthetic into a relatively vascular plane. In a study on 12 patients undergoing major abdominal surgery and bilateral TAP block with $40 \mathrm{ml}$ of lidocaine $1 \%$, the highest concentration of lidocaine $(5.5 \mu \mathrm{g} / \mathrm{ml})$ was recorded $15 \mathrm{~min}$ after the block and results indicate that a TAP block can potentially cause systemic toxicity of a local anesthetic. ${ }^{38}$ Griffiths et al. ${ }^{39}$ reported that the mean peak total venous ropivacaine concentrations exceeded a potentially neurotoxic threshold value $(2.2 \mathrm{mg} / \mathrm{mL})$ after bilateral TAP block with $3 \mathrm{mg} / \mathrm{kg}$ ropivacaine; ${ }^{39}$ moreover, he described a case of neurotoxicity from local anaesthetic with TAP block in a woman undergoing to Caeserean section. ${ }^{40}$ Scherrer et al. ${ }^{41}$ described a case of cardiotoxicity due to overdose of ropivacaine after a TAP block realized by anaesthesiologist followed to a wound infiltration realized by surgeon. ${ }^{41}$

Some authors described the addition of dexamethasone, an efficient glucocorticoid drug with anti-inflammatory properties, to prolong effect of local anaesthetics. ${ }^{42}$ In according to several works, ${ }^{43,44}$ we suggest use of ropivacaine solution (a local anesthetic that provides a longer postoperative analgesia, with a greater margin of safety for cardiotoxicity and neurotoxicity) $2 \mathrm{mg} / \mathrm{ml}$ or $3.75 \mathrm{mg} / \mathrm{ml}$ with a volume of maximum $20 \mathrm{ml}$ for each side for adult patients, avoiding to exceed dose of $2 \mathrm{mg} / \mathrm{kg}$, thus measuring anaesthetic dose to weight patient. More studies occurred to evaluate the role and potential of dexamethasone or other adjuvants.

\section{TAP block and wound infiltration}

In order to treat postoperative pain, administer less opioid drugs and use local anaesthetic to modulate pain response, infiltration wound is an older technique than TAP block, applying in many surgeries: a Cochrane review identified a number of studies showing a reduction of cumulative morphine consumption at $48 \mathrm{~h}$ by $25-55 \%$ with continuous wound infusion of the local anaesthetic, ${ }^{45}$ same results have obtained in breast surgery. ${ }^{46}$

Although some articles suggest a superiority of local wound infiltration rather than TAP block in Caesarean section, ${ }^{8}$ cholecystectomy ${ }^{47}$ or radical prostatectomy ${ }^{48}$ in term of pain reduction 
in the first postoperative 4-6 hours, an interesting meta-analysis of Guo et al. ${ }^{49}$ including nine studies with a total of 500 patients undergoing several abdominal surgeries, showed that TAP block provides superior analgesia compared with wound infiltration in the setting of a multimodal analgesic regimen in term of opioid consumption and pain at rest and after coughing; subgroup analysis indicated that adults may have benefits additional to the analgesic effect than children. The PONV incidence and sedation scores were not significant difference between TAP block and wound infiltration in most included studies. Furthermore, no serious complications were reported following two groups in all nine studies. ${ }^{49}$

\section{TAP block and epidural analgesia}

Thoracic epidural analgesia (TEA) can be considered the gold standard for postoperative analgesia in major abdominal surgeries via laparotomy; however, there are well-known side effects and potentially catastrophic risks to this technique as well as a reported failure rate ranging from $17 \%$ to $37 \%$ of cases: a systematic review including 20000 patients showed an incidence of premature catheter dislodgement of 5.7\% ${ }^{50}$ TEA may cause an important motor block involving lower limbs after several hours too, precluding the possibility of a quick recovery of walking and urinary function; ${ }^{51}$ hypo and hyper-function is strictly related to surgery outcomes, as demonstrated by Sugimoto, ${ }^{52}$ regarding a poor pain control in first two postoperative days, hypotension events, oliguria, risk of central nervous system (CNS) and systemic infection, dural puncture or epidural haematoma. ${ }^{53}$ There are not differences between TEA and IV analgesia with opioid with regard to proportion of patients who experienced a delay in gastrointestinal recovery, fluid bolus requirements within the first three perioperative days, rates of infection, pulmonary complications. ${ }^{54}$

In last decade, many authors compared effects of epidural analgesia and TAP block: in 70 patients undergoing laparoscopic bowel resections, randomized to be treated with continuous epidural or continuous four-quadrant TAP block analgesia, Niraj et al. ${ }^{55}$ did not found any difference in median visual analogue scores (VAS) during coughing at 24 hours after surgery and tramadol consumption between two groups, with a significant reduction of the time of removal of the urinary catheter ( 72 versus 44 hours).

In a previous non-inferiority trial in 62 patients undergoing hepatobiliary and renal surgeries (bilateral subcostal TAP block with boluses of bupivacaine versus continuous epidural analgesia) results are different: the TAP catheter technique had a therapeutic failure rate of $30 \%$ compared with $22 \%$ in the epidural group; failure in the TAP catheter group was mainly associated with uncontrolled pain from the lateral margin of transverse incisions extending beyond the anterior axillary line; moreover, the epidural group had a success rate of $78 \%$, while the TAP catheter group's is of $63 \%$. In the other hand, complications with epidural catheters included two accidental dural punctures and epidural filter disconnection in two patients but there were no complications with TAP catheters. ${ }^{56}$

In a recent study including 50 patients undergoing open abdominal surgery via laparotomy (continuous bilateral TAP block versus continuous epidural analgesia) comparable results have obtained about postoperative control pain at rest and on coughing in the first 24 hours and patient satisfaction..$^{57}$ A randomized trial enrolling 42 patients undergoing abdominal surgery via laparotomy treated with continuous TAP block or continuous TEA resulted in similar effects in term of pain and consumption of postoperative fentanyl..$^{58}$
Wahba and Kamal $\mathrm{SM}^{59}$ compared these two techniques in 44 American Society of Anesthesiologists physical status (ASA) III and ischemic heart disease patients undergoing laparotomic surgeries: TEA group showed a reduction of morphine consumption ( 7 versus $11.5 \mathrm{mg}$ in the first day), a reduced time of first morphine request (311 versus $210 \mathrm{~min}$ ), but incidence of hypotension events was higher in TEA group.

Another trial compared intravenous PCA with morphine, TEA and TAP block in laparoscopic high anterior resection: use of TAP blocks was associated with significant reduction in mean morphine usage at 12 hours and 24 hours, significant difference was seen on days 4 and 5 between the TAP block group and the epidural group after most of the epidural catheters were removed, while cumulative opioid use was significantly lower for the epidural group at all time points up to discharge than for the PCA group and it was significantly lower up to 72 hours than for the TAP block patients; hospital length of stay was shorter in TAP group (4 days) than in TEA group (6 days). ${ }^{60}$

These studies demonstrated that TAP block analgesia is safer than TEA, above all in patients in which TEA is not viable (patients with coagulation disorders, severe malformations of the spine, infection of puncture site or septicaemia, severe hypovolemia): TAP block may realize on patients with severe coagulation disorders, with a particular attention to visualize eventual blood vessels during the US guided puncture, like epigastric artery rami in subcostal TAP; the only absolute contraindication is infection or presence of surgical incision and drainages in site of puncture. Also adverse effects are more frequent during TEA: dural puncture, epidural haematoma, hypotension events, motor block, urinary retention, pruritus do not occur in TAP block. However, unsuccessful rate of continuous TAP block analgesia is higher than TEA, because catheter dislodgement and positioning is more frequent for two or four different catheters on abdomen wall, with a consequent request of more attention during postoperative management, above all during patient movements.

On the other hand, efficacy of TEA is commonly the same or superior than TAP block, in term of on rest and on movement postoperative pain and opioid use reduction, with less adverse effects, such as nausea and vomiting; but other works, with a greater number of patient, are necessary to evaluate the real superiority of TEA on TAP block in term of pain reduction but also adverse effects and patient satisfaction. Certainly, TEA and TAP block are better than intravenous PCA analgesia alone.

In conclusion, we suggest use of epidural analgesia only if patient conditions allow it and a strict catheter's control after surgery is possible, especially regard to management of epidural catheter and eventual administration of anticoagulants therapy and prophylaxis. We prefer continuous TAP block only in selected cases (collaborative patients, with trained ward nurse and colleagues); for other patients, one-shot TAP block with a long duration anaestetic drug, as ropivacaine, at the start of surgery is the optimal solution. Obviously, these techniques are only a part of multimodal analgesia, that includes a PCA administration of opioid drug, acetaminophen and/or NSAIDs, if possible.

\section{Conclusion}

Nowadays, the key role of TAP block in a multimodal model of analgesia is clear. The importance of postoperative pain control is at the center of discussions about implementation of Enhanced Recovery After Surgery (ERAS) pathways in many hospitals worldwide as standard perioperative protocols above all bowel resections surgery, 
with consequent earlier recovery of gastrointestinal function, a shorter hospital stay, low readmissions and re-hospitalization rates and finally low management cost for hospitals. With optimization of pre-hospitalization and postoperative protocols, surgical laparoscopic techniques, intraoperative and postoperative fluid management, helping of a team of different professional roles (surgery, anaesthesiologist, nurse, nutritionist), a faster recovery of patients is possible and recommended.

In this scenery, different studies proposed the application of TAP block analgesia to meliorate standard ERAS protocols, that encourages a multimodal analgesia model with use of local or peripheral analgesia for a opioid-sparing regime: a shorter in-hospital length of stay (four versus six days) with a less re-hospitalization rate has demonstrated in laparoscopic colorectal surgery within ERAS protocol. ${ }^{61} \mathrm{~A}$ larger retrospective study showed advantages of introduction of TAP block respect to intravenous PCA analgesia in ERAS protocol in term of in-hospital length of stay. ${ }^{62}$ In a recent pilot study on 100 patients undergoing laparoscopic colorectal resections, the addition of the preincision bilateral TAP block to classical intravenous PCA analgesia reduces postoperative pain in PACU, after 24 hour, patient satisfactory and in-hospital length of stay. ${ }^{63}$

algesia and its versatility in every type of abdominal surgery, through laparotomic and laparoscopic via: it reduces complication rate of opioid use, improves state-of-life of patients in first two days, allowing a faster recovery of bowel function without reduction of respiratory function, reduces length of in-hospital stay after surgery and corresponding costs.

The choice of TAP block instead of another analgesia technique, as TEA, must be applied by the anaesthesiologist evaluating each case, the right cost-benefit ratio for patient, the possibility of a careful postoperative, the desirable multidisciplinary pain management, as part of a more complex pathway which aims at full recovery of ability of the patient as soon as possible.

\section{Acknowledgments}

None.

\section{Conflicts of Interset}

None.

\section{References}

1. Rafi AN. Abdominal field block: a new approach via the lumbar triangle. Anaesthesia. 2001;56(10):1024-1026.

2. Rozen WM, Tran TM, Ashton MW, et al. Refining the Course of the Thoracolumbar Nerves: A New Understanding of the Innervation of the Anterior Abdominal Wall. Clin Anat. 2008;21(4):325-333.

3. Hebbard P, Fujiwara Y, Shibata Y, et al. Ultrasound-guided transversus abdominis plane (TAP) block. Anaesth Intensive Care. 2007;35(4):616-617.

4. Farooq M, Carey M. A case of liver trauma with a blunt regional anesthesia needle while performing transversus abdominis plane block. Reg Anesth Pain Med. 2008;33(3):274-275.

5. McDonnell JG, O'Donnell B, Curley G, et al. The Analgesic Efficacy of Transversus Abdominis Plane Block After Abdominal Surgery: A Prospective Randomized Controlled Trial. Anesth Analg. 2007;104(1):193-197.

6. Niraj G, Searle A, Mathews M, et al. Analgesic efficacy of ultrasoundguided transversus abdominis plane block in patients undergoing open appendectomy. Br J Anaesth. 2007;103(4):601-605.
7. Heil JW, Ilfeld BM, Loland VJ, et al. Ultrasound-guided transversus abdominis plane catheters and ambulatory perineural infusions for outpatient inguinal hernia repair. Reg Anesth Pain Med. 2010;35(6):556-558.

8. Kassir R, Tiffet O, Bourbon M, et al. Laparoscopic Hernia Repair With 3-Millimeter Instruments: A Point of Technique and Illustrative Case Video. Surg Innov. 2015;22(4):366-367.

9. Telnes A, Skogvoll E, Lonnée H. Transversus abdominis plane block vs. wound infiltration in Caesarean section: a randomised controlled trial. Acta Anaesthesiol Scand. 2015;59(4):496-504.

10. Deborah SK, Bridget OE, Conor PD. Demonstrating the Benefits of Transversus Abdominis Plane Blocks on Patient Outcomes in Laparoscopic Colorectal Surgery: Review of 200 Consecutive Cases. $J$ Am Coll Surg. 2014;219(6):1143-1148

11. Brady RR, Ventham NT, Roberts DM, et al. Open transversus abdominis plane block and analgesic requirements in patients following right hemicolectomy. Ann R Coll Surg Engl. 2012;94(5):327-330.

12. Bhattacharjee. Transversus abdominis plane block in abdominal hysterectomy. Journal of Anaesthesiology Clinical Pharmacology. 2014;30(3):391-396.

13. Elkassabany N, Ahmed M, Malkowicz SB, et al. Comparison between the analgesic efficacy of transverses abdominis plane (TAP) block and placebo in open retropubic radical prostatectomy: a prospective, randomized, double-blinded study. J Clin Anesth. 2013;25(6):459-465.

14. Aniskevich S, Taner CB, Perry DK, et al. Ultrasound-guided transversus abdominis plane blocks for patients undergoing laparoscopic handassisted nephrectomy: a randomized, placebo-controlled trial. Local and Reg Anesth. 2014;7:11-16.

15. Mohammadi SS, Dabir A, Shoeibi G. Efficacy of Transversus Abdominis Plane Block for Acute Postoperative Pain Relief in Kidney Recipients: A Double-Blinded Clinical Trial. Pain Med. 2014;15(3):460-464.

16. Hotujec BT, Spencer RJ, Donnelly MJ, et al. Transversus abdominis plane block in robotic gynecologic oncology: A randomized, placebocontrolled trial. Gynecologic Oncology. 2015;136(3):460-465.

17. Heil JE, Nakanote KA, Madison SJ, et al. Continuous Transversus Abdominis Plane (TAP) Blocks for Postoperative Pain Control after Hernia Surgery: A Randomized, Triple-Masked, Placebo-Controlled Study. Pain Med .12014;5(11):1957-1964.

18. Gomez-Rios MA, Paech MJ. Continuous posterior TAP analgesia after laparoscopic colorectal surgery. Anaesthesia. 2014;69(9):1051-1064.

19. Fredrickson MJ, Seal P. Ultrasound-guided transversus abdominis plane block for neonatal abdominal surgery. Anaesth Intensive Care. 2009;37(3):469-472.

20. Sammons G, Ritchey W. Use of Transversus Abdominis Plane (TAP) Blocks for Pain Management in Elderly Surgical Patients. AORN J. 2015;102(5):493-497.

21. Hebbard PD, Saidman, Lawrence. Subcostal transversus abdominis plane block under ultrasound guidance. Anesth \& Analg. 2008;106(2):674-675

22. Hebbard PD, Barrington MJ, Vasey C. Ultrasound-Guided Continuous Oblique Subcostal Transversus Abdominis Plane Blockade Description of Anatomy and Clinical Technique. Reg Anesth Pain Med. 2009;35(5):436-441.

23. Børglum J, Jensen K, Christensen AF, et al. Distribution patterns, dermatomal anesthesia, and ropivacaine serum concentrations after bilateral dual transversus abdominis plane block. Reg Anesth Pain Med. 2012;37(3):294-301.

24. Tran TMN, Ivanusic JJ, Hebbard PD, et al. Determination of spread of injectate after ultrasound-guided transversus abdominis plane block: a cadaveric study. Br J Anaesth. 200;102(1): 123-127. 
25. Bhatia N, Arora S, Wig J, et al. Comparison of posterior and subcosta approaches to ultrasound-guided transverse abdominis plane block for postoperative analgesia in laparoscopic cholecystectomy. J Clin Anesth. 2014;26(4):294-299.

26. Shin HJ, Oh AY, Baik JS, et al. Ultrasound-guided oblique subcostal transversus abdominis plane block for analgesia after laparoscopic cholecystectomy; a randomized, controlled, observer-blinded study. Minerva Anestesiol. 2014;80(2):185-193.

27. Wu Y, Liu F, Tang H, et al. The Analgesic Efficacy of Subcostal Transversus Abdominis Plane Block Compared with Thoracic Epidural Analgesia and Intravenous Opioid Analgesia After Radical Gastrectomy. Anesth Analg. 2014;117(2):507-513

28. Albrecht E, Kirkham KR, Endersby RV, et al. Ultrasound-Guided Transversus Abdominis Plane (TAP) Block for Laparoscopic GastricBypass Surgery: a Prospective Randomized Controlled Double-Blinded Trial. Obes Surg. 2013;23(8):1309-1314.

29. Milan ZB, Duncan B, Rewari V, et al. Subcostal Transversus Abdominis Plane Block for Postoperative Analgesia in Liver Transplant Recipients. Transplant Proc. 2014;43(7):2687-2690.

30. Serag Eldin M, Mahmoud F, El Hassan R, et al. Intravenous patientcontrolled fentanyl with and without transversus abdominis plane block in cirrhotic patients post liver resection. Local Reg Anesth. 2014;7:27-37.

31. Basaran B, Basaran A, Kozanhan B, et al. Analgesia and Respiratory Function after Laparoscopic Cholecystectomy in Patients receiving Ultrasound-Guided Bilateral Oblique Subcostal Transversus Abdominis Plane Block: A Randomized Double-Blind Study. Med Sci Monit. 2014;21:1304-1312.

32. Borlgum, Jensen K, Christensen AF, et al. Distribution Patterns, Dermatomal Anesthesia, and Ropivacaine Serum Concentrations After Bilateral Dual Transversus Abdominis Plane Block. Reg Anesth Pain Med. 2012;37(3):294-301.

33. HebbardPD. TAPblocknomenclature. Anaesthesia. 2015;70(1):112-113.

34. Takimoto S, Sakai N, Ono M. The effect of adding upper and lower subcostal transversus abdominis plane blocks to lateral transversus abdominis plane block after laparoscopic cholecystectomy. Eur J Anaesthesiol. 2015;32 (11):819-820.

35. Lee TH, Barrington MJ, Tran TM, et al. Comparison of extent of sensory block following posterior and subcostal approaches to ultrasoundguided transversus abdominis plane block. Anaesth Intensive Care. 2010;38(3):452-460.

36. Carney J, Finnerty O, Rauf J, et al. Studies on the spread of local anaesthetic solution in transversus abdominis plane blocks. Anaesthesia. 2011;66(11):1023-1030

37. Moeschler SM, Murthy NS, Hoelzer BC, et al. Ultrasound-guided trans versus abdominis plane injection with computed tomography correlation: a cadaveric study. J Pain Res. 2013;6:493-496.

38. Kato N, Fujiwara Y, Harato M, et al. Serum concentration of lidocaine after transversus abdominis plane block. J Anaesth. 2009;23(2):298-300.

39. Griffiths JD, Barron FA, Grant S, et al. Plasma ropivacaine concentration after ultrasound guided transverses abdominis plane block. Br J Anaesth 2010;105(6):853-856

40. Griffiths JD, Le NV, Grant S, et al. Symptomatic local anaesthetic toxicity and plasma ropivacaine concentrations after transversus abdominis plane block for caesarean section. Br J Anaesth. 2013;110(6):996-1000.

41. Scherrer V, Compere V, Loisel C, et al. Cardiac arrest from local anesthetic toxicity after a field block and transversus abdominis plane block: a consequence of miscommunication between the anesthesiologist and surgeon. A Case Rep. 2013;1(5):75-76.
42. Akkaia A, Yildiz I, Tekelioglu UY, et al. Dexamethasone added to levobupivacaine in ultrasound-guided tranversus abdominis plain block increased the duration of postoperative analgesia after caesarean section: a randomized, double blind, controlled trial. Eur Rev Med Pharmacol Sci. 2014;18(5):717-722.

43. Simpson D, Curran MP, Oldfield V, et al. Ropivacaine. A review of its use in regional anaesthesia and acute pain management. Drugs. 2005;65(18):2675-2717.

44. Abdul RM, Yahya N, Sulaiman O, et al. Comparing the effectiveness of ropivacaine $0.5 \%$ versus ropivacaine $0.2 \%$ for transabdominis plane block in providing postoperative analgesia after appendectomy. Acta Anaesthesiologica Taiwanica. 2014;52(2):49-53.

45. Bamigboye AA, Hofmeyr GJ. Local anaesthetic wound infiltration and abdominal nerves block during caesarean section for postoperative pain relief. Cochrane Database Syst Rev. 2002;3:CD006954.

46. Rosaeg OP, Bell M, Cicutti NJ, et al. Pre-incision infiltration with lidocaine reduces pain and opioid consumption after reduction mammoplasty. Reg Anesth Pain Med. 1998;23(6):575-579.

47. Ortiz J, Suliburk JW, Wu K, et al. Bilateral transversus abdominis plane block does not decrease postoperative pain after laparoscopic cholecystectomy when compared with local anesthetic infiltration of trocar insertion sites. Reg Anesth Pain Med. 2012;37(2):188-192.

48. Skjelsager A, Ruhnau B, Kistorp TK, et al. Transversus abdominis plane block or subcutaneous wound infiltration after open radical prostatectomy: A randomized study. Acta Anaesthesiol Scand. 2013;57(4):502-508.

49. Qingduo Guo, Rui Li, Lixian W, et al. Transversus abdominis plane block versus local anaesthetic wound infiltration for postoperative analgesia: a systematic review and meta-analysis. Int J Clin Exp Med. 2015;8(10):17343-17352.

50. Dolin SJ, Cashman JN, Bland JM. Effectiveness of acute postoperative pain management: I. Evidence from published data. Br J Anaesth. 2002;89(3):409-423.

51. Scott DA, Chamley DM, Mooney PH, et al. Epidural ropivacaine infusion for postoperative analgesia after major lower abdominal surgery-a dose finding study. Anesth Analg. 1995;81(5):982-986.

52. Sugimoto M, Nesbit L, Barton JG, et al. Epidural anesthesia dysfunction is associated with postoperative complications after pancreatectomy. $J$ Hepatobiliary Pancreat Sci. 2015;23(2):102-109.

53. Landoni $\mathrm{G}$, Isella $\mathrm{F}$, Greco $\mathrm{M}$, et al. Benefits and risks of epidural analgesia in cardiac surgery. Br J Anaesth. 2015;115(1):25-32.

54. Winer AG, Sfakianos JP, Puttanniah VG, et al. Comparison of perioperative outcomes for epidural versus intravenous patientcontrolled analgesia after radical cystectomy. Reg Anesth Pain Med. 2015;40(3):239-244.

55. Niraj G, Kelkar A, Hart E, et al. Comparison of analgesic efficacy of four-quadrant transversus abdominis plane (TAP) block and continuous posterior TAP analgesia with epidural analgesia in patients undergoing laparoscopic colorectal surgery: an open-label, randomised, noninferiority trial. Anaesthesia. 2014;69(4):348-355.

56. Niraj G, Kelkar A, Jeyapalan A, et al. Comparison of analgesic efficacy of subcostal transversus abdominis plane blocks with epidural analgesia following upper abdominal surgery. Anaesthesia. 2011;66(6):465-471.

57. Ganapathy S, Sondekoppam RV, Terlecki M, et al. Comparison of efficacy and safety of lateral-to-medial continuous transversus abdominis plane block with thoracic epidural analgesia in patients undergoing abdominal surgery: A randomised, open-label feasibility study. Eur J Anaesthesiol. 2015;32(11):797-804 
58. Rao Kadam V, Van Wijk RM, Moran JI, et al. Epidural versus continuous transversus abdominis plane catheter technique for postoperative analgesia after abdominal surgery. Anaesth Intensive Care. 2013;41(4):476-481.

59. Wahba SS, Kamal SM. Analgesic efficacy and outcome of transversusabdominis plane block versus low thoracic-epidural analgesia after laparotomy in ischemic heart disease patients. $J$ Anesth. 2014;28(4):517-523.

60. Ris F, Findlay JM, Hompes R, et al. Addition of transversus abdominis plane block to patient controlled analgesia for laparoscopic high anterior resection improves analgesia, reduces opioid requirement and expedites recovery of bowel function. Ann R Coll Surg Engl. 2014;96(8):579-585.
61. Favuzza J, Brady K, Delaney CP. Transversus abdominis plane blocks and enhanced recovery pathways: making the 23-h hospital stay a realistic goal after laparoscopic colorectal surgery. Surg Endosc. 2013;27(7):2481-2486.

62. Favuzza J, Delaney CP. Outcomes of Discharge after Elective Laparoscopic Colorectal Surgery with Transversus Abdominis Plane Blocks and Enhanced Recovery Pathway. J Am Coll Surg. 2013;217:503-506.

63. Keller DS, Tahilramani RN, Flores-Gonzalez JR, et al. Pilot study of a novel pain management strategy: evaluating the impact on patient outcomes. Pilot study of a novel pain management strategy: evaluating the impact on patient outcomes. Surg Endosc. 2015. 